\title{
Regulatory Effects of WRAP53 on Radiosensitivity of Laryngeal Squamous Cell Carcinoma Cells
}

\author{
Hui Qiu, De-Ying Zhao, Li-Mei Yuan, Gong Zhang, Cong-Hua Xie*
}

\begin{abstract}
Background: Telomere length is closely associated with cellular radiosensitivity and WRAP53 is required for telomere addition by telomerase. In this research we assessed radiosensitivity of laryngeal squamous cell carcinoma Hep-2 cell lines after WRAP53 inhibition, and analyzed the molecular mechanisms. Materials and Methods: phWRAP53-siRNA and pNeg-siRNA were constructed and transfected into Hep-2 cells with lipofectamine. Expression of WRAP53 was analyzed by RT-PCR and Western-blottin, radiosensitivity of Hep-2 cells was assessed colony formation assay, and the relative length of telomeres was measured by QPCR. $\underline{\text { Results: The data }}$ revealed that the plasmid of phWRAP53-siRNA was constructed successfully, and the mRNA and protein levels of WRAP53 were both obviously reduced in the Hep-2 cell line transfected with phWRAP53-siRNA.After Hep-2 cells were irradiated with $X$-rays, the $D_{0}$ and $S_{2}$ were 2.481 and 0.472 , respectively, in the phWRAP53-siRNA group, much lower than in the control group $\left(\mathrm{D}_{0}\right.$ and $\mathrm{SF}_{2}$ of 3.213 and 0.592$)(P<0.01)$. The relative telomere length in the phWRAP53-siRNA group was $0.185 \pm 0.01$, much lower than in the untreated group $(0.523 \pm 0.06)$ and the control group $(\mathbf{0 . 4 3 5} \pm \mathbf{0 . 0 1})$. Conclusions: Decreasing the expression of WRAP53 using RNA interference technique can enhance the radiosensitivity of Hep-2 cell lines by influencing the telomere length. WRAP53 is expected to be a new target to regulate the radiosensitization of tumor cells.
\end{abstract}

Keywords: WRAP53 - Hep-2 - laryngeal squamous cell carcinoma - radiosensitivity - telomere length

Asian Pac J Cancer Prev, 16 (7), 2975-2979

\section{Introduction}

Radiation therapy (RT) is an important kind of treatment in head and neck squamous cell carcinoma (HNSCC) patients, especially to the patients with locally advanced disease. In the treatment of laryngeal squamous cell carcinoma, many studies have demonstrated organpreserving induction definitive radio-therapy (RT) is an alternative with comparable outcomes to laryngectomy. Although most of patients with laryngeal squamous cell carcinoma can acquire satisfactory curative effect, a few patients have poor outcome due to resistance to radiation. It is reported that the radiation resistance almost accounts for $50 \%$ in all patients of treatment failure with laryngeal squamous cell carcinoma (Arshad et al., 2014; Megwalu et al., 2014; Mok et al., 2014).

As a kind of new human telomerase holoenzyme protein, WRAP53 is required for telomere addition by telomerase. Depletion of WRAP53 in cells significantly inhibited telomere elongation, but not reduced telomerase activity (Venteicher et al., 2009; Zhong et al., 2011; Stern et al., 2012; Megwalu et al., 2014). Telomere and telomerase play an important role in occurrence and development of malignancy. Telomere length is closely associated with cellular radiosensitivity, the longer of the telomeres means that the tumor cells are more resistible to radiation (Fairlie et al., 2015; Laster et al., 2015).

To explore the effect of WRAP53 on the tumor cells, in this study, we regulated WRAP53 expression by gene silencing technology, observed the effect on the radio-sensitivity of human Hep- 2 cells and analyzed the molecular mechanism.

\section{Materials and Methods}

\section{Cell line and cell culture}

Human laryngeal squamous cell carcinoma cell line Hep-2 was purchased from cell collection centre of Xiangya School of Medicine, Central South University. The cells were cultured in RPMI 1640 medium (HyClone, USA) supplemented with $10 \%$ fetal bovine serum (FBS). The cells were incubated at $37^{\circ} \mathrm{C}$ in humidified atmosphere containing $5 \% \mathrm{CO}_{2}$, and the medium was changed every 3 days. To keep the cells at optimal proliferating conditions, they were passaged at $80 \%$ confluence and seeded at $20 \%$ confluence. 


\section{siRNA design and construction}

siRNAs directed against WRAP53 mRNA (named as phWRAP53-siRNA) were designed and constructed by Shanghai GenePharma Co. Ltd., China. Each pair of siRNA was designed to have two complementary oligonucleotide chains, and it was verified that there was no homology with another human gene by BLAST search. The phWRAP53-siRNA sequence: 5'-GUGUGGGUGAGCUGAUAUATT3' (sense) and 5'-UAUAUCAGCUCACCCACACTT-3' (antisense); Negative-siRNA sequence: 5'-UUCUCCGAACGUGUCACGUTT-3' (sense) and 5'-ACGUGACACGUUCGGAGAATT-3' (antisense).

\section{Cell transfection}

The experiments were divided into three groups: Hep2, Hep-2/pNeg-siRNA and Hep-2/ phWRAP53-siRNA. Twenty-four hours before cell transfection experiments, $2.5 \times 105$ cells were seeded in 6-well plate in RPMI 1640 without antibiotics, corresponding to a density of $60 \%$ $70 \%$ at the time of transfection. $4 \mu \mathrm{l}$ siRNA and $8 \mu \mathrm{l}$ Lipofectamine $^{\mathrm{TM}} 2000$ (Invitrogen USA) were mixed respectively with DMEM without FBS and incubated for $5 \mathrm{~min}$ at room temperature. Then the reagents were combined and incubated for $20 \mathrm{~min}$ before adding to the cells in DMEM without FBS. After transfection, cells were cultivated in standard condition for 4-6 $\mathrm{h}$, then fresh RPMI 1640 with $10 \%$ FBS was added. As a control, the pNegsiRNA was used with the same transfection conditions.

\section{RT-PCR detection}

The cells after transfection were collected after 48 $\mathrm{h}$, and the total RNA was extracted by Trizol reagent (Fermentas LTU) according to the manufacturer's protocol. $1 \mu$ lotal RNA were reversed by RevertAid ${ }^{\mathrm{TM}}$ M-MuLv Reverse Transcriptase (Fermentas LT), the RT reaction was performed at $42^{\circ} \mathrm{C}$ for $60 \mathrm{~min}$, then $70^{\circ} \mathrm{C}$ for $5 \mathrm{~min} .1 \mu \mathrm{l}$ of $\mathrm{RT}$ reaction product was as the template of PCR. The primer sequences of WRAP53 were 5'-CTACAACCACCTGGATGAGC-3' (upstream) and 5'-TAACCAGACTGCCGGAGA-3' (downstream), the length of amplification was $396 \mathrm{bp}$ and the annealing temperature was $60^{\circ} \mathrm{C}$. The primer sequences of GAPDH, an internal control, were 5'-TTAGCACCCCTGGCCAAGG-3' (upstream) and 5'-CTTACTCCTTGGAGGCCATG-3' (down-stream), the length of amplification was $396 \mathrm{bp}$ and the annealing temperature was also $60^{\circ} \mathrm{C}$.The PCR reactions were run in triplicate at $94^{\circ} \mathrm{C}$ for $5 \mathrm{~min}$, followed by 30 cycles at $94^{\circ} \mathrm{C}$ for $30 \mathrm{sec}$, and $72^{\circ} \mathrm{C}$ for $1 \mathrm{~min} .10 \mu \mathrm{l}$ of $25 \mu \mathrm{l}$ total PCR products were separated by electrophoreses on $1.5 \%$ agarose gel containing ethidium bromide and photographed under automatic gel imaging system.

\section{Western-blot analysis}

The whole cell proteins were extracted by using protein extraction reagent. Protein concentration was detected by using a BCA protein assay kit according to the manufacturer's protocol. The same amount of total protein (40-50 $\mu \mathrm{g})$ from each lysate was loaded onto $10 \%$ SDS-polyacrylamide gel. After electrophoresis, separated proteins were transferred to nitrocellulose membranes, and then they were rinsed with TBS-T $(0.1 \mu \mathrm{mol} / \mathrm{L}$ Tris- $\mathrm{HCl}$, $\mathrm{pH} 7.5 ; 0.9 \%$ sodium chloride; $0.05 \%$ tween 20 ) for 15 min and blocked with powdered non-fat milk for $1.5 \mathrm{~h}$ at $37^{\circ} \mathrm{C}$. And then proteins were incubated with mouse antihuman monoclonal antibodies against WRAP53 (Abgent, USA, 1:1000 dilution), or $\beta$-actin (Abgent, USA, 1:1000 dilution) at $4^{\circ} \mathrm{C}$ overnight.

After washing with TBS-T for $4 \times 10 \mathrm{~min}$, nitrocellulose membranes were incubated with horseradish peroxidase (HRP)-labeled goat anti-mouse second antibodies (Sigma, USA, 1:3000 dilution) for $2 \mathrm{~h}$ at room temperature and washed with TBS-T for $4 \times 10 \mathrm{~min}$. Nitrocellulose membranes were detected specificly by using ECL method (Pierce, USA).

\section{Fluorescence quantitative PCR analysis}

Genomic DNA was extracted from transfected cells by standard procedures, and stored at $4^{\circ} \mathrm{C}$ at a concentration of $250 \mathrm{ng} / \mu \mathrm{l}$. Genomic DNA was diluted into distilled water just prior to setting up QPCR runs. The telomere and 36B4 (an internal control) primer sequences were synthetized according to the reference (Cawthon et al., 2002). The telomere primer sequences were 5' -GGTTTT TGAGGGTGAGGGTGAGGGTGAGGGTGAGGGT-3' (tel1) and 5'-TCCCGACTATCCCTATCCCTATCCCTA TCCCTA-3' (tel2); The 36B4 primer sequences were 5'-CAGCAAGTGGGAAGGTGTAATCC-3' (36B4u) and 5'-CCCATTCTATCATCAACGGGTACAA-3' (36B4d). The thermal cycling profile of QPCR reactions were run at $95^{\circ} \mathrm{C}$ for $5 \mathrm{sec}$, followed 30 cycles of $95^{\circ} \mathrm{C}$ for $15 \mathrm{sec}$, and $54^{\circ} \mathrm{C}$ for $2 \mathrm{~min}$. For each experimental sample the ratio of these dilution factors is the relative telomere to single copy gene $(\mathrm{T} / \mathrm{S})$ ratio. So the relative telomere length, that is the relative $\mathrm{T} / \mathrm{S}$ ratio (T/S of one sample relative to the $\mathrm{T} / \mathrm{S}$ of another sample) is $2^{-\triangle \Delta \mathrm{ct}}$, and $\triangle \triangle \mathrm{ct}$ $=\left(\triangle \mathrm{ct}^{\text {telomere }}-\triangle \mathrm{ct}^{36 \mathrm{~B} 4}\right)$.

\section{Colony formation assay}

Cells at exponential phase of growth were plated in triplicate onto 6-well plate at the required concentration and allowed to attach overnight. Hep-2, Hep-2/pNegsiRNA, and Hep-2/phWRAP53-siRNA cells were exposed to $0,1,2,4,6,8$ and 10 Gy X-ray $(6 \mathrm{MV}, \mathrm{SSD}=80 \mathrm{~cm}$, $1.205 \mathrm{cGy} / \mathrm{s})$. Then the cells were cultured for 10 to 14 days at $37^{\circ} \mathrm{C}$ in $5 \% \mathrm{CO}_{2}$ to get violet colonies. Colonies stained with $0.5 \mathrm{ml}$ of $0.5 \%$ crystal violet solution for 20 min and counted by using a microscope $(\times 40)$. A viable colony was defined as having at least 50 cells after 10 to 14 days of growth. The colonies were counted from each triplicate sample and presented as average values. The surviving fraction of treated cells was normalized to the plating efficiency of unirradiation cells.

Cell survival was plotted as a function of dose and fitted using the single-hit multi-hit model ( $\mathrm{SF}=1-\left(1-\exp ^{-}\right.$ $\left.\left.{ }^{\mathrm{kD}}\right)^{\mathrm{N}}\right)$ and the linear-quadratic model $\left(\mathrm{SF}=\exp \left(-\alpha \mathrm{D}-\beta \mathrm{D}^{2}\right)\right)$, where $\mathrm{SF}$ is the cell survival, $\mathrm{D}$ is the dose of radiation, $\alpha$ and $\beta$ were constants and $\mathrm{SF}_{2}$ was surviving fraction at 2 Gy which was calculated from the actual data, and SER was defined as the radio of phWRAP53-siRNA group SF and pNeg-siRNA group SF at $2 \mathrm{~Gy}$. 
Statistical analysis

SPSS 13.0 software was used for all statistical procedures. All data were presented as means \pm standard deviation $( \pm \mathrm{s})$. A two-tailed unpaired t test was used to compare the statistical significance of the differences in data from the two groups and $\chi^{2}$ test was used to count data. Differences at $p<0.05$ were considered significant.

\section{Results}

The expression of WRAP53 mRNA after transfection

The expression of WRAP53 mRNA was detected by RT-PCR (Figure 1). There was no marked difference of WRAP53 mRNA between Hep-2 and Hep-2/pNeg-siRNA, while the WRAP53 mRNA of Hep-2/phWRAP53-siRNA was inhibited markedly. The internal control GAPDH expression was consistent in three cell lines.

The expression of WRAP53 protein after transfection

Western-blot analysis (Figure 2) showed that there was no difference of WRAP53 protein between Hep-2 and Hep-2/pNeg-siRNA, while the expression of WRAP53 protein of Hep-2/phWRAP53-siRNA was decreased obviously. The internal control $\beta$-actin had no change in three cell lines.

Radiation biology parameters $\left(D_{0}, D q\right.$ and $\left.S F_{2}\right)$ were detected by colony formation assay

The cell survival curves that was obtained by linearquadratic model and single-hit multi-hit model (Figure 3 , Figure 4) shown that $\mathrm{D}_{0}, \mathrm{Dq}$ and $\mathrm{SF}_{2}$ values (Table 1) of Hep-2/phWRAP53-siRNA were all lower than that

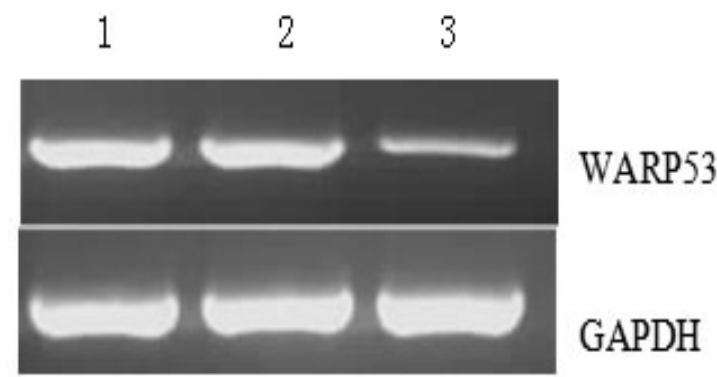

Figure 1. RT-PCR Analysis of Expression of WRAP53 mRNA in Hep-2 Cells. 1: Hep-2; 2: Hep-2/pNeg-siRNA; 3 : Hep-2/phWRAP53-siRNA

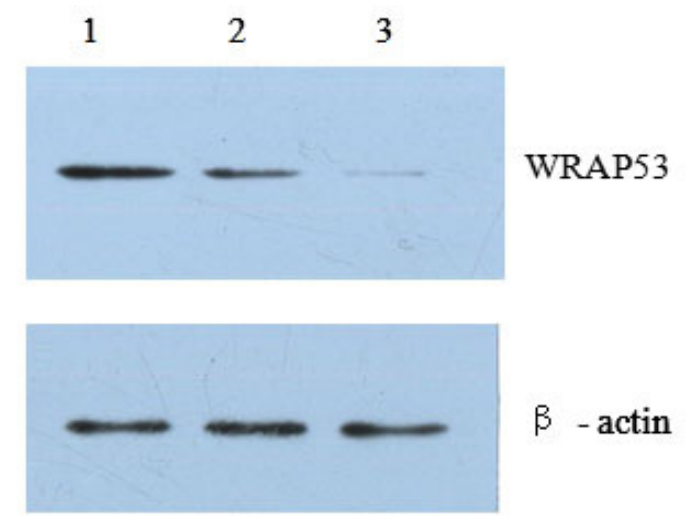

Figure 2. Western-blot Analysis of Expression of WRAP53 Protein in Hep-2 Cells. 1: Hep-2; 2: Hep-2/ pNeg-siRNA; 3: Hep-2/phWRAP53-siRNA

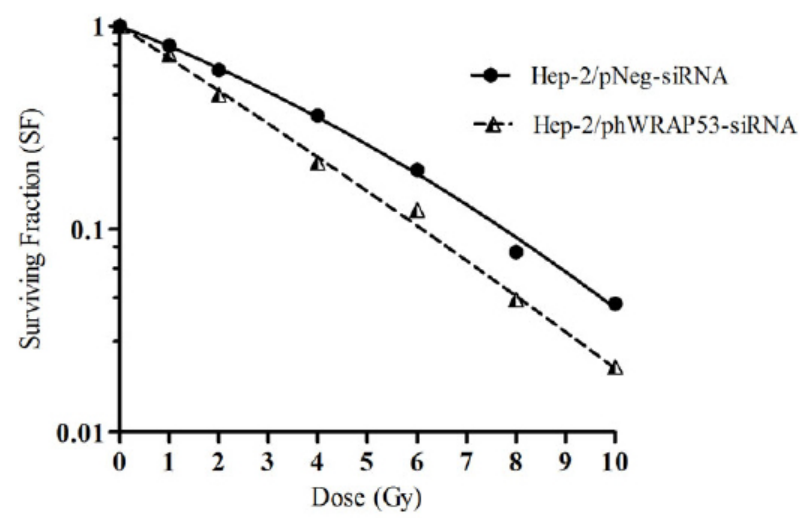

Figure 3. The Survival Curve of Hep-2 Cells Fitted by Linar Quadratic (L-Q) Model

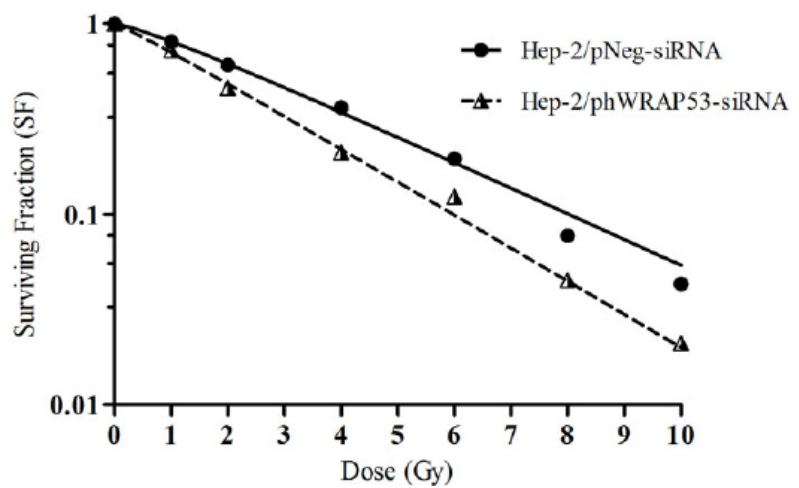

Figure 4. The Survival Curve of Hep-2 Cells Fitted by One-hit Multi-target Model

Table 1. Radiosensitivity Parameters of Hep-2 Cells Transfected with siRNA

\begin{tabular}{lcccc}
\hline groups & $\mathrm{D}_{0}(\mathrm{~Gy})$ & $\mathrm{Dq}(\mathrm{Gy})$ & $\mathrm{SF}_{2}{ }^{*}$ & $\mathrm{SER}$ \\
\hline Hep-2/pNeg-siRNA & 3.213 & 0.311 & 0.592 & \\
Hep-2/phWRAP53-siRNA & 2.481 & 0.111 & 0.472 & 1.29 \\
\hline
\end{tabular}

$* p<0.01$

Table 2. QPCR Analysis of Amplification of DNA36B4 and Telomere in Hep-2 cells

\begin{tabular}{lcc}
\hline groups & $\begin{array}{c}\text { DNA36B4 } \\
\triangle \text { ct value }\end{array}$ & $\begin{array}{c}\text { telemere } \\
\triangle \text { ct value }\end{array}$ \\
\hline Hep-2 & $17.76 \pm 0.25$ & $18.57 \pm 0.60$ \\
Hep-2/pNeg-siRNA & $17.21 \pm 0.08$ & $18.44 \pm 0.16$ \\
Hep-2/phWRAP53-siRNA & $17.17 \pm 0.08$ & $19.52 \pm 0.67$ \\
\hline
\end{tabular}

of Hep-2/pNeg-siRNA, statistical analysis showed that the difference between the two groups was significant, $\mathrm{P}<0.01$. Meanwhile the SER of Hep-2/phWRAP53-siRNA was 1.290. All these data meant that phWRAP53-siRNA increased the cellular radiosensitivity of Hep-2 cells.

The relative telomere length after $6 M V X$-ray irradiation

The $\triangle$ ct value of amplification of DNA36B4 and telomere in three cells line were obtained by QPCR analysis (Table 2). Then the relative T/S value of each cell line was calculated according to the $\triangle \mathrm{ct}$ value and formula

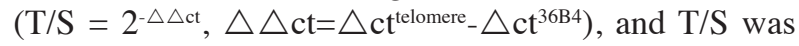
positively correlated with the relative telomere length. The result shown that the T/S value of Hep-2 and Hep-2/pNegsiRNA was $0.435 \pm 0.01$ and $0.523 \pm 0.06$, respectively, the two cell lines were no significant difference. While the $\mathrm{T} / \mathrm{S}$ value in the phWRAP53-siRNA was $0.185 \pm 0.01$, 


\section{Qiu Hui et al}

which were much lower than those of control, all these data meant that phWRAP53-siRNA shorten the telomere length of Hep-2.

\section{Discussion}

The study of radiation sensitizing is a hot question in radiation oncology field. The purpose of radiation sensitizing is to improve intrinsic radiosensitivity of tumor cells to ionizing radiation and enhance the radiation effect to cancer cells, especially to irradiated resistant cells.

Telomere plays an important role in radiosensitivity of carcinoma cells, because it can be used as the hallmarks of radiosensitivity (Fairlie et al., 2015). It is reported that telomere length is inversely correlates with radiosensitivity in human carcinoma cells with the same tissue background or the different tissue background. Telomerase is a ribonucleoprotein complex which maintains the length and integrity of telomeres for continuous cell proliferation in cancer. The telomerase activity is expressed in about $90 \%-95 \%$ of human cancer cells or $60 \%-70 \%$ of the immortalized human cells $(\mathrm{Hu}$ et al., 2014). WRAP53 protein is found to be a new component of telomerase, and it plays a crucial role in regulating telomere synthesis by telomerase. Depletion of WRAP53 in cells significantly inhibited telomere elongation, but not influenced telomerase activity. Thus, we consider that WRAP53 may become an effective target for radiotherapy sensitization of laryngeal squamous cell carcinoma Cell (Freund et al., 2014).

In this study, RNAi technique is used to inhibit the WRAP53 mRNA and protein expression or activity of Hep-2 cell lines to observe the cellular radiosensitivity. RNA interference is one of the most active hot spots in molecular biology research. RNAi has the characteristic of highly sequence specificity, high-efficiency and action quick. Very small amounts of siRNA can specifically inhibit the expression of certain gene in cancer cells. The expression of WRAP53 mRNA and protein were inhibited obviously after being transfected with the plasmid of phWRAP53-siRNA (Figure 1 and Figure 2), and there are no difference in another cell lines. These show that WRAP53-targeted siRNA inhibited WRAP53 specifically.

After $6 \mathrm{MV} \mathrm{X}$-ray irradiation, Colony formation assay shown that the $\mathrm{SF}_{2}$ of Hep-2/phWRAP53-siRNA was lower than those of control cells, which elucidated that

Hep-2/phWRAP53-siRNA was more sensitive to radiation than Hep-2/pNeg-siRNA, the same result was acquired by QPCR. Meanwhile, the telomere length was shorter than those of control cells. We consider that after inhibition of WRAP53, telomerase could not synthesize telomere effectively and Double Strand Breaks (DSB) induced by irradiation could not be repaired through NHRJ repair pathway, which accelerated proliferating death of cells and increased the cellular radiosensitivity.

In order to further understand whether the cellular radiosensitivity was increased by affecting telomere, the telomere length was tested after inhibition of WRAP53. At present, the detection medthods of telomere length are Southern-blot, flow cytometry-fluorescent in situ hybridization (Flow-FISH), hybridization protection
assay(HPA), telomere amount and length assay(TALA). Among them, the Southern-blot is the most widely used and classic method, but its application was restricted by more operating steps and higher experimental requirements. Cawthon RM created a method to detect the relative telomere length by using quantitative PCR (QPCR) in 2002. In QPCR, the ratio of telomere (T) repeat copy number to single copy gene $(\mathrm{S})$ copy number is doubtless, and $\mathrm{T} / \mathrm{S}$ is positively correlated with the mean telomere lengths. So when we obtained the relative T/S value of each sample according to the $\triangle \mathrm{ct}$ value and formula $\left(\mathrm{T} / \mathrm{S}=2^{-\triangle \Delta \mathrm{ct}}, \triangle \Delta \mathrm{ct}=\Delta \mathrm{ct}^{\text {telomere }}-\triangle \mathrm{ct}^{36 \mathrm{~B} 4}\right)$, we can know the relative telomere length. As the $\mathrm{T} / \mathrm{S}$ value and Southern-blotting have a good correlation, some scholars have adopted this method to detect the relative telomere length of large sample now. In this study, we amplified telomere and single copy gene 36B4, and then obtained the T/S value. By comparison, we found that phWRAP53siRNA shorten the telemere length of Hep-2 cells. So we think telomere shortening may be a molecular mechanism that WRAP53 affects cellular radiosensitivity.

Other researches have suggested that WRAP53 expressed mainly in carcinoma tissue, including laryngeal squamous cell carcinoma tissues, Mahmoudi $\mathrm{S}$ found that the expression of WRAP53 in cancer cells is 20 times as much as in normal cells. In addition, some studies have demonstrated the polymorphism of WRAP53 gene was associated with breast cancer risk, and regulated the biological activities of P53 (Sedaie et al., 2014; SaldanaMeyer et al., 2014). Sun found that enhanced WRAP53 expression was observed in HNSCC with low intrinsic radiosensitivity and low expression was observed in cells with high intrinsic radiosensitivity, that is to say, it prognosticates that WRAP53 may be an ideal target for enhancing radiosensitivity (Sun et al., 2014). Our results obtained from this research also confirmed this hypothesis and provided a new way to increase the radiosensitivity of laryngeal squamous cell carcinoma. In future, WRAP53 might be useful as a prognostic biomarker and a potential target for the diagnosis and therapy of head and neck carcinomas.

\section{References}

Arshad H, Jayaprakash V, Gupta V, et al (2014). Survival differences between organ preservation surgery and definitive radiotherapy in early supraglottic squamous cell carcinoma. Otolaryngol Head Neck Surg, 150, 237-44.

Cawthon RM (2002). Telomere measurement by quantitative PCR. Nucleic Acids Res, 30, 47.

Fairlie J, Harrington L (2015). Enforced telomere elongation increases the sensitivity of human tumour cells to ionizing radiation. DNA Repair, 25, 54-9.

Freund A, Zhong FL, Venteicher AS, et al (2014). Proteostatic control of telomerase function through TRiC-mediated folding of TCAB1. Cell, 159, 1389-403.

Hu Y, Bobb D, Lu Y, et al (2014). Effect of telomerase inhibition on preclinical models of malignant rhabdoid tumor. Cancer Genet, 207, 403-11.

Laster BH, Isaacson C, Perets E, et al (2015). Keeping those telomeres short! an innovative intratumoral long-term drug delivery system. J Cancer Res Clin Oncol, 141, 23-34.

Mahmoudi S, Henriksson S, Farnebo L (2011). WRAP53 

promotes cancer cell survival and is a potential target for cancer therapy. Cell Death Dis, $2,114$.

Megwalu UC, Sikora AG (2014). Survival outcomes in advanced laryngeal cancer. JAMA Otolaryngol Head Neck Surg, 140, 855-60.

Mok G, Gauthier I, Jiang H, et al (2014). Outcomes of intensitymodulated radiotherapy versus conventional radiotherapy for hypopharyngeal cancer. Head Neck, [Epub ahead of print].

Saldaña-Meyer R, Gonzalez-Buendia E, Guerrero G, et al (2014). CTCF regulates the human p53 gene through direct interaction with its natural antisense transcript, Wrap53. Genes Dev, 28, 723-34.

Sedaie Bonab A, Pouladi N, Hosseinpourfeizi MA, et al (2014). Single-strand conformational polymorphism analysis of a common single nucleotide variation in WRAP53 gene, rs2287499, and evaluating its association in relation to breast cancer risk and prognosis among Iranian-Azeri population. Med Oncol, 31, 168.

Stern JL, Zyner KG, Pickett HA, et al (2012). Telomerase recruitment requires both TCAB 1 and Cajal bodies independently. Mol Cell Biol, 32, 2384-95.

Sun CK, Luo XB, Gou YP, et al (2014). TCAB1: a potential target for diagnosis and therapy of head and neck carcinomas. Mol Cancer, 13, 180.

Venteicher AS, Artandi SE (2009). TCAB1: driving telomerase to Cajal bodies. Cell Cycle, 8, 1329-31.

Zhong F, Savage SA, Shkreli M, et al (2011). Disruption of telomerase trafficking by TCAB1 mutation causes dyskeratosis congenita. Genes Dev, 25, 11-6. 